\title{
Modeling, Identification and Control at Telemark University College
}

\author{
B. Lie, D. Di Ruscio, R. Ergon, B. Glemmestad \\ M. Halstensen, F. Haugen, S. Mylvaganam, N.-O. Skeie, D. Winkler
}

Telemark University College, P.O. Box 203, N-3901 Porsgrunn, Norway

\begin{abstract}
Master studies in process automation started in 1989 at what soon became Telemark University College, and the 20 year anniversary marks the start of our own PhD degree in Process, Energy and Automation Engineering. The paper gives an overview of research activities related to control engineering at Department of Electrical Engineering, Information Technology and Cybernetics.
\end{abstract}

Keywords: modeling, simulation, identification, control, sensor technology

\section{Introduction}

The Norwegian research journal MIC was initiated by late Professor Jens Glad Balchen, with the first issue published in 1980. MIC has played a central role in Norwegian cybernetics research, as it coincided with a dramatic growth in the number of $\mathrm{PhD}$ students and gave these an arena to publish. Telemark University College (HiT) salutes the journal, and those who made the journal possible.

The master studies in engineering at HiT started in 1988, and the initial board was led by Finn Lied and included Inge Johansen and Sven G. Terjesen, all central people in the engineering community of Norway in the last part of the 20th century. The leader of the engineering studies was May-Britt Hägg, now professor at The Norwegian University of Science and Technology (NTNU). In 1989, a study in Process Automation started; this study was planned by Terje Hertzberg, Steinar Sælid, Gudolf Kjærheim, Sven G. Terjesen, Ivar Loe, Jens I. Ytreeide, and Rolf Ergon. Later, Ytreeide became professor in these studies, while Loe was adjunct professor for many years. The Process Automation study was led by Rolf Ergon, now professor emeritus. In 1994, these studies became part of HiT, organized under Faculty of Technology (HiT-TF).
From the start, the MSc studies in Porsgrunn had their accreditation from the Ministry of Education and Research, while the PhD study was formally a degree at NTNU, where HiT-TF operated almost as a faculty under NTNU. In April 2009, the Ministry of Education and Research gave HiT the right to give our own PhD-degree, in Process, Energy, and Automation Engineering.

The current MSc studies are in Process Technology, Systems and Control Engineering, and Energy and Environmental Technology, and they are taught in English. Initially, the strong position of the regional process industry shaped the process automation study, which had a strong emphasis on modeling of dynamic systems, numeric methods, process chemistry, separation technology, thermodynamics, etc. Control engineering was also important, with topics in multivariable control, optimal and predictive control, state estimation, and control structures for industrial processes. Instrumentation technology and process safety were core topics, and laboratory exercises widely used.

With a compact group of teachers in close touch with the students, this enabled necessary changes in pace with the developments in the regional and national industry, and today, the core topics are modeling of dynamic systems, model based control, model based 
sensor technology, and industrial IT. The content of the study is thus more general today, but the theory is still tested through laboratory work as well as examples and projects/theses which to a significant degree (70-80\%) come from the regional industry.

Although the terms modeling, identification and control (MIC) do not explicitly mention instrumentation and sensor technology (IST), we will still consider IST a part of MIC: without IST, there is no information to be used in identification and control. And without models, there is no IST: as an example, consider the mercury thermometer - clearly it is not the temperature that is measured, but the expansion of mercury; the temperature is inferred from a model of the relationship between temperature and expansion.

The paper is organized as follows. In Section 2, glimpses into education and research activities are given, while in Section 3, a survey of past and on-going $\mathrm{PhD}$ studies is given. In section 4 , an overview of activities in societies is given.

\section{Glimpses into Education and Research Activities}

\subsection{Introduction}

Throughout the years, Department of Engineering Cybernetics at NTNU has had an important influence on the faculty: Ytreeide laid a foundation in instrumentation technology and system safety, and emphasizing practical laboratory work. Professor Bernt Lie has worked mainly with control relevant mechanistic models in the process industry and application of such models in MPC, Associate Professor David Di Ruscio has focused on advanced control and in particular on the development of subspace methods within system identification, and Associate Professor Finn Haugen has worked with basic and practical control engineering with implementation in process data systems. Associate Professor Nils-Olav Skeie adds a dimension with work in industrial IT and soft-sensors.

Professor Emeritus Rolf Ergon has brought inspiration from Chalmers University of Technology and servo technology, while the strong Nordic chemometrics society has lent ideas through Adjunct Professor Kim Esbensen, Associate Professor Maths Halstensen, and Ergon. A further influence is from the Technische Universität Berlin, via Professor Saba Mylvaganam and his work in sensor technology and the fusion of models and sensor systems, and Assistant Professor Dietmar Winkler and his work in modeling and simulation of electric drives as well as simulation languages such as Modelica.
Professor Bjørn Glemmestad did his BSc and MSc at HiT, and his PhD study at NTNU in association with HiT; he recently came from the process industry with experience in application of nonlinear MPC.

\subsection{Control Education and Laboratory Experience}

We believe that students get a much deeper understanding of theoretical methods by implementing the methods in practical applications. To this end, we have developed a number of laboratory assignments which are part of different courses in our master study in Systems and Control Engineering. We have standardized on using PCs (laptops or desktops) with National Instruments LabVIEW and the inexpensive NI USB 6008 $\mathrm{I} / \mathrm{O}$ device, but MATLAB and SIMULINK are also used to some extent Haugen (2005, 2008); Haugen et al. (2007, 2008). As lab stations we use air heaters (seven items) Haugen (2009a) and water tanks (six items) Haugen (2009i), which are "desktop" lab stations. Due to the large number of lab stations, we can run labs in parallel, and with small student groups. Although the final aim in the assignments is to apply the solutions developed by the students to the physical system, the students are required to apply their solutions to simulated processes first. The feedback from the students on these assignments is very positive.

The following laboratory assignments have been designed:

- Implementing an industrial PID controller and a measurement filter from scratch as $\mathrm{C}$ code with practical features such as bumpless transfer, anti integral windup, and reverse/direct action. The controller is applied to either the water tank or the air heater. Haugen (2009b,h,d).

- Hardware-in-the-loop simulator based on an industrial PID controller (Fuji PGX) controlling a simulated process. Haugen (2009h,c).

- Soft-sensor (state estimator) for estimating an unknown outflow from a water tank using various methods: "Direct estimator" (solving the model for the unknown variable), a Luenberger observer, and a Kalman filter. The flow estimate is used in feed-forward control of the water level. Haugen (2009h,f).

- System identification of air heater in the form of a discrete-time transfer function using a subspace identification method ( $\mathrm{n} 4 \mathrm{sid}$ in MATLAB). A temperature controller for the simulated process 
is tuned in SIMULINK, and a practical temperature control system is then implemented in LabVIEW. Haugen (2009h,g).

- Model-based predictive control (MPC) of air heater, using the MPC controller of LabVIEW Haugen (2009h,e).

\subsection{Sensor Data Fusion, Soft Sensors and Sensor Networking}

"Data fusion is a process of associating, correlating, combining measured data and other relevant information from single and/or multiple sensors to achieve better estimates of observed parameters or even estimating parameters normally not amenable for direct measurements. Data fusion gives an added leverage to the measurement and control engineer in achieving more complete and timelier assessments of process status indicating simultaneously undesirable or dangerous situations, and their significance. The fusion process involves continuous refinements of its estimates and assessments, and by evaluation of the need for additional sources of information (i.e., possibly new sensors), leading very often to the modification of the process itself, leading thus to an overall improvement of the process and its performance indicators", Viumdal et al. (2010).

Data fusion is inherently associated with the concept of soft sensors. Soft sensor or virtual sensor is a common name for software based algorithms processing/fusing a plethora of measurements. The fusion of these measurements can be used in the estimation of new quantities that need not or can not be measured. Strategies based on soft sensors are essential in modern data fusion and use among others the following:

- Statistical Methods

- Kalman filters

- Artificial Neural Networks (ANN)

- Fuzzy Logic

- Principal Component Analysis

- Markov Models

- System identification methods

- Support vector machines

- Fuzzy Neural Methods

In the R\&D activities, usually carried out in collaboration with industries and research institutes, the focus is on process measurements and sensorics, with innovations based on new usage of existing sensors, incorporating new sensors and developing algorithms for soft sensors. Some recent applications are in
- powder technology, Wærstad et al. (2002); Mylvaganam (2003); Datta et al. (2003); Mylvaganam et al. (2003); Mylvaganam and Dyakowski (2005); Datta et al. (2007a,b)

- light metal industries, Viumdal et al. (2010)

- production, storage and transport of oil and gas, Vefring et al. (2002), Nygaard et al. (2004a,b); Nygaard and Nrevdal (2006); Nygaard et al. (2006, 2007), Lorentzen et al. (2008)

- patient care on $24 / 7$ basis

- soft sensor approach to diagnosis of electrical machines, sensors and actuators, Yahoui et al. (2004); Yahoui and Mylvaganam (2009)

Process tomography is essentially a form of sensor networking and data fusion on a smaller scale and involves multimodal, multifunctional sensors, data from which have to be fused to give insight into the process, preferably non-intrusively, Alme (2006.); Alme and Mylvaganam (2006b,a), Alme and Mylvaganam (2007). As such, process tomography involves all the above and requires electromagnetic modeling, to which some of our R\&D efforts are dedicated to, Lorentzen et al. (2008); Timmerberg et al. (2009).
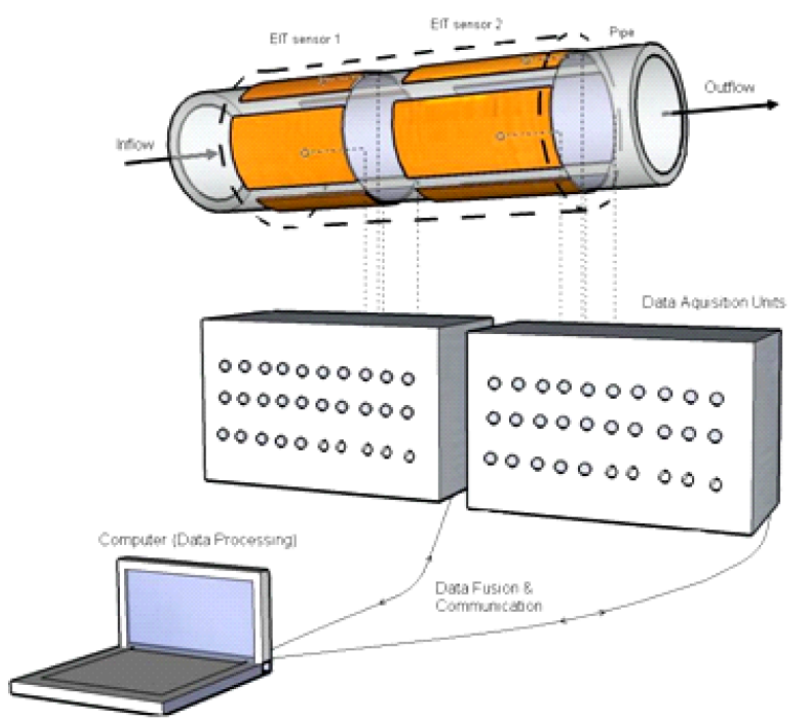

Figure 1: Sensor Data Fusion/ Soft Sensors involved in Process Tomography with multiple resistive and capacitive sensors serves simultaneously as an example of sensor networking when different protocols are used, in selecting the combinations of sensors and their automatic switching. Graphic by PhD student Yan Ru. 


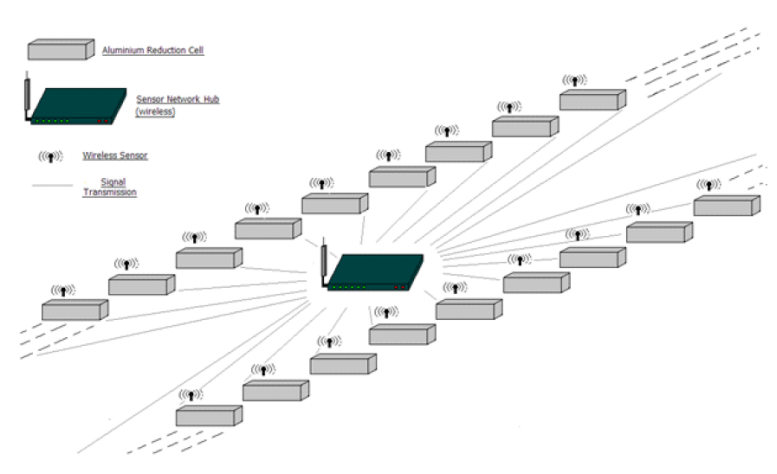

Figure 2: Sensor Networking in Light metal Industries, involving innovative usage of existing and new sensors. Graphic by PhD student Yan $\mathrm{Ru}$.

\subsection{Applied Chemometrics}

Acoustic chemometrics is a relatively new method for on-line process monitoring based on characterisation of system vibrations as generated by an industrial process such as a manufacturing process or transportation flow. Acoustic chemometrics is applicable for quantitative analysis of constituents for process monitoring and for physical characterisation of the state of process equipment. Principal component analysis (PCA) or partial least squares regression (PLS) Martens and Næs (1989); Esbensen (2001) based on empirical data are used to extract relevant information from relevant acoustic signals. The PLS model can then be used to predict parameters of interest based on new independent acoustic spectra. Proper validation of resulting regression models is critical in order to develop realistic prediction models for industrial process monitoring.

The main advantage of acoustic chemometrics compared with many other on-line methods for process monitoring is the use of nonintrusive so-called "clampon" sensors which can be easily mounted onto the process equipment (pipelines, reactors etc.). The sensor, which often is a standard accelerometer or a so-called acoustic emission (AE) sensor, has no moving parts, and can withstand harsh environments. The measured acoustic signatures will often contain information about several process-relevant properties which makes it possible to predict several parameters/states from the same acoustic spectrum.

Acoustic emission from industrial processes is often considered as audible noise only, but it has recently been proven that within this "noise" there is also a significant part of useful information Esbensen et al. (1998); Halstensen et al. (1998); Esbensen et al. (1999); Halstensen and Esbensen (2000); Halstensen et al. (2006), which can be used for processes mon- itoring. The fact that almost all processes produce some kind of acoustic emission opens up the potential for applications which depend totally on sound, signal processing, sensor technology and multivariate calibration.

Several papers covering multiphase fluids, system state of rotating machinery and powder characterisation have been published Esbensen et al. (1998); Halstensen et al. (1998); Esbensen et al. (1999); Halstensen and Esbensen (2000); Halstensen et al. (2006) reporting that this method is a promising on-line process analytical technology (PAT) approach.

Acoustic chemometrics has its greatest benefits in cases where traditional sensors and measurement techniques, such as flow, temperature and pressure transmitters can not be used. In many cases it is preferable to use nonintrusive sensors because their counterpart may cause disturbances, e.g., fouling and clogging inside the process equipment such as pipelines, reactors cyclones etc. Figure 3 shows an overview of the data path from acoustic emission to the final multivariate calibration model.

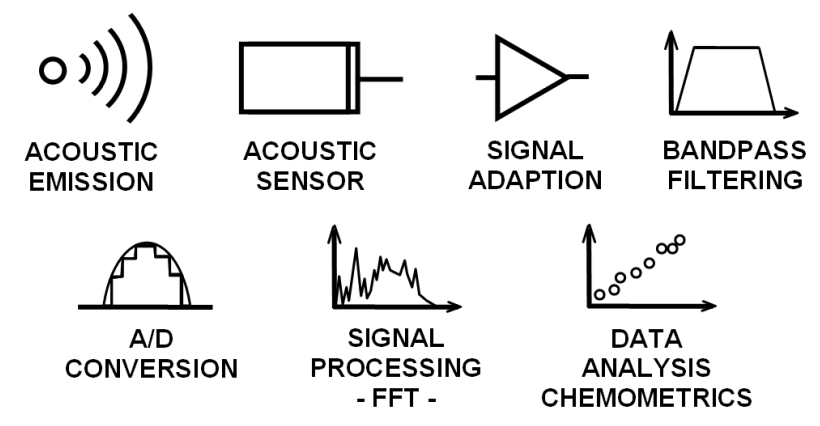

Figure 3: Schematic overview of acoustic chemometrics.

The main research activities of the Acoustic Chemometrics Research Group are acoustic process monitoring, multivariate image analysis, chemometric theory, multivariate process monitoring, representative sampling, and soft sensors.

\subsection{Soft Sensors for Level Estimation in Oil/Water Separators}

Separation of oil, water and gas is an important process stage in oil and gas production. Such mixed fluids with different densities are often separated using a gravity separator. An unwanted emulsion will develop in the layer between oil and water. The level and thickness of the emulsion layer together with the oil and water content is therefore one of the important properties when controlling the oil output flow rate of 
the separator. No perfect measurement system exists for oil/water separators today. The research work at HiT is based on combining a set of pressure sensor devices, absolute or relative, and a guided radar sensor device to estimate the oil, emulsion and water contents in the separator. The method is based on a number of standard and inexpensive sensor devices and data fusion strategies like the Partial Least Squares Regression (PLSR), Principal Component Regression (PCR), and Artificial Neural Networks (ANN) in developing data driven models for estimation of the level and thickness of the oil, emulsion, and water content. The work shows that it is possible to combine a set of pressure sensor devices, a guided radar sensor device, and models calibrated using PCR, PLSR, or ANN to estimate the liquid level, the water level, the thickness of the emulsion layer, and the thickness of the oil layer in the oil/water separator Skeie et al. (2006); Skeie and Lie (2006); Skeie (2008). The advantage of this method compared to existing methods are: inexpensive, simple to install, independent of foam on top of the oil layer, redundancy of sensor devices and does not expose people to any harmful radiation. Further work will investigate how the number and type of sensor devices will influence among others the accuracy and robustness of such soft sensors, and how the models can be calibrated depending on the locations of the sensor devices and the density of the liquids.

\subsection{Theoretical Aspects of Process Monitoring}

Theoretical issues in system identification and chemometrics, with regards to process monitoring applications, has been and still is an active research area at HiT. Product quality estimation based on known process inputs and secondary process measurements was investigated by Ergon and Di Ruscio (1997), and an approach based on identification of an output error (OE) model using a prediction error method was developed by Ergon (1999b). Not only is an OE model necessary, it also makes it possible to use low rate and even irregular sampling data of the primary quality variables, Ergon and Halstensen (2001), which is quite important from a practical point of view. This system identification approach can also be combined with multivariate calibration methods from chemometrics, Ergon (1999a).

A second problem investigated by Ergon is multivariate calibration model reduction. The projection based principal component regression (PCR) and partial least squares regression (PLSR) methods for static process data often result in more than two principal components, and process monitoring based on traditional score and loading plots is then a non-attractive option. This can be solved by further projections, such that the relevant process information can be presented in a single score-loading-contribution plot Ergon (2002b), Ergon (2003, 2004, 2006, 2009a). As part of the model reduction effort, the highly profiled, patented and popular orthogonal signal correction method OPLS Trygg and Wold (2002) has also been studied. Although this is claimed to be a pre-processing method, it has been shown that it in fact is a disguised post-processing procedure Ergon (2005). It can also be shown that even further model reduction is possible Ergon (2007).

A third problem that has been investigated is a residual inconsistency resulting from the conventional NIPALS algorithm used in PLSR. This problem was first found by Ergon as a by-product of another work Ergon (2002a), but at the time judged to be of little practical interest. However, the related and essential problem of score-loading correspondence for the modeled data was investigated, Ergon (2002b). A later paper Pell et al. (2007) brought attention to the problem with the twenty year old and very central algorithm, and recommended use of the Bidiag2 algorithm Golub and Kahan (1965) instead of NIPALS, and this caused a heated debate in the chemometrics community. Ergon (2009b) clarified that the problem could be solved by a simple re-interpretation of the NIPALS results, and Ergon, Halstensen and Esbensen are in an upcoming paper looking further into the problem in relation to squared prediction errors in the process monitoring context. This problem is illustrated in Figure 4, where $\mathrm{SPE}_{\mathrm{C}}=\varepsilon_{\mathrm{C}}^{T} \varepsilon_{\mathrm{C}}$ based on the conventional PLSR residual $\varepsilon_{\mathrm{C}}$ may both over- and underestimate the true squared perpendicular distance $\mathrm{SPE}_{\mathrm{B}}=\varepsilon_{\mathrm{B}}^{T} \varepsilon_{\mathrm{B}}$ from a sample $\mathbf{z}$ to the projection space where the scores are found.

The results are the projection $\hat{\mathbf{z}}_{\mathrm{B}}$ and the non-orthogonal mapping $\hat{\mathbf{z}}_{\mathrm{C}}$, while the orthogonal complement of the column space of $\mathbf{W}$ defines the common residual space. The prediction coefficient vector $\hat{\mathbf{b}}$ is contained in the column space of the loading weight matrix $\mathbf{W}$, while an alternative (and never used) projection $\hat{\mathbf{z}}_{\mathrm{P}}$ is contained in the column space of the loading matrix $\mathbf{P}$. Points $\mathrm{A}$ and $\mathrm{B}$ refer to an example in the manuscript of the upcoming paper.

\subsection{Process Monitoring Based on Wireless Sensor Network}

There is an increasing focus on and interest in wireless communication and services utilizing this concept. Process monitoring is part of the research work at HiT and wireless communication with and within measurement systems is part of this research area. Process 


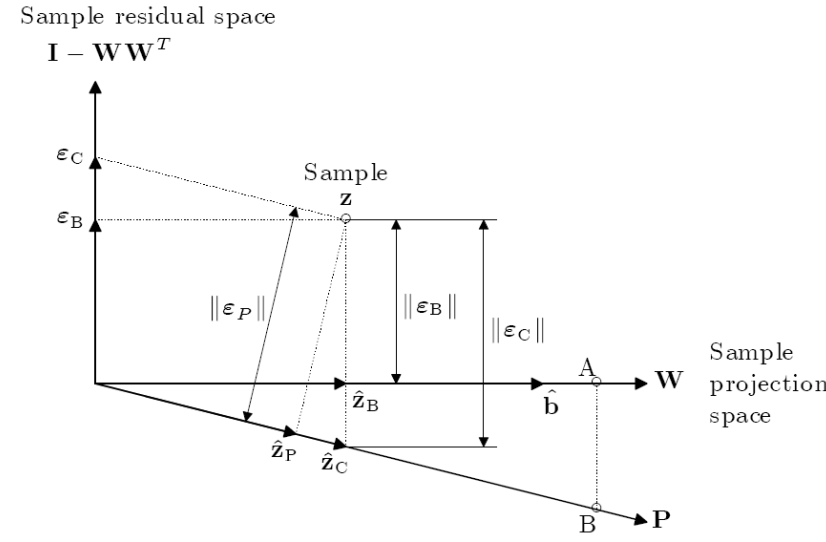

Figure 4: Orthogonal splitting of sample $\mathbf{z}$ into $\hat{\mathbf{z}}_{\mathrm{B}}$ and $\varepsilon_{\mathrm{B}}$ based on the Bidiag2 algorithm, and nonorthogonal splitting into $\hat{\mathbf{z}}_{\mathrm{C}}$ and $\varepsilon_{\mathrm{C}}$ based on the conventional NIPALS algorithm.

monitoring can be based on a network of sensors, and this network can be a wireless network of sensor nodes. Such a wireless sensor network (WSN) will have one or a few gateways where external systems can connect to get information from the WSN. One of the areas for the research work at HiT is development of virtual sensors (soft sensors) using the WSN as a sensor array where the number of devices and the type of measurement devices can change dynamically. The virtual sensor will be the connection point between any software applications and the WSN and should optimize the usage of the WSN as regards the number of sensor nodes, the information in the network, redundancy and the power usage in the nodes. Other areas of research is the usage of soft sensors inside the WSN to reduce the network traffic and the power usage and how WSN can be utilized in Building Automation Systems (BAS) to adapt such systems to the usage of the buildings.

HiT is one of the academic partners in the Center for Wireless Innovation (CWI) (www.cwin.no), which is a facilitator for industry and the academic participants in forming a strategic partnership in wireless $\mathrm{R} \& \mathrm{D}$.

\subsection{Modelling and Simulation of Electrical Systems}

The simulation of systems is a very useful method to investigate different behaviors of physical systems, e.g., stress-tests, faults. Thus we can test if a certain experiment will damage the test equipment or even worse might prove to have dangerous impacts on personnel. Especially fault scenarios are the ideal application field for simulation runs. We would like to know what happens if certain devices fail and perhaps derive security measures which will protect our applications if a certain fault occurs.

In the past it was often good enough to simulate different aspects of a physical system individually within their physical domains. For each of those domains there was a specialized simulation tool. This becomes problematic when different physical systems interact which each other, as it is normally the case in the real world. Now one has to find some means to couple different tools in a way that they can exchange simulation results during run-time (aka co-simulation). This is normally quite inflexible wrt. step-size and solvertype.

Another solution is to find a simulation language which allows for modelling of different physical domains within the same language and tool. The nonproprietary modeling language Modelica ${ }^{\circledR}$ (ModelicaAssociation (2009)) was especially developed to simplify the simulation in different physical domains within one simulation model and also have the means of exchanging your models without being bound by a particular tool. In addition, the non-profit organization Modelica Association provides a standard library (Modelica Standard Library) that already contains a huge amount of components and connectors from different domains. This freely down-loadable library also serves as a common base for different tools (free and commercial types are available Modelica.org (2009)).

The multi-domain capability allows us to easily build simulation models of complex systems including, for example, mechanical, electrical, and chemical components and reactions. Furthermore it allows us to concentrate on the physics of a model rather than building models which represent mathematical equations which in turn then represent the actual physical behavior.

Using Modelica allows us to build models of systems like electric drives/generators where we have to deal with mechanical, electrical, and thermal quantities. These can also be extended to even more complex models including mechanical or electrical faults Winkler and Gühmann (2008, 2009). Such systems can then be analyzed and optimized wrt. physical meaningful results. An example would be to optimize the control of the voltage level in a weak electricity network.

\subsection{System Identification}

\subsubsection{The Relation to the Partial Least Squares (PLS) Algorithm}

The Partial Least-Squares (PLS) algorithm has received widespread attention and is widely used in Chemometrics, which has been defined as The use of mathematics and statistics on chemical data, Martens and Næs (1989). In our view the PLS method is com- 
plicated to understand due to the iterative nature of computing the solution, $B_{\mathrm{PLS}}$, for the regression coefficients, $B$, in a linear (or bi-linear) model $Y=X B+E$ from known data matrices $X \in \mathbb{R}^{N \times r}$ and $Y \in \mathbb{R}^{N \times m}$. In Di Ruscio (2000), insight and theoretical understanding into the Partial Least Squares algorithm is given, and a new, non-iterative formulation of the PLS algorithm is given in case of univariate data (PLS1), i.e., $m=1$ and $Y$ a column vector. In that paper it is shown that the PLS1 algorithm is equivalent to using a truncated Cayley-Hamilton polynomial expression of degree $1 \leq a \leq r$ for the matrix inverse $\left(X^{T} X\right)^{-1}$ $\in \mathbb{R}^{r \times r}$ which is used to compute the Least Squares (LS) solution. Here the integer $a$ is the number of PLS components. Furthermore, the $a$ coefficients, $p \in \mathbb{R}^{a}$, in this polynomial are computed as the optimal LS solution (minimizing parameters) to the prediction error. Hence, the PLS1 solution is optimal in the sense that $p^{*}=\arg \min _{p}\left\|Y-X K_{a} p\right\|_{F}^{2}$, where then $B_{\mathrm{PLS}}=$ $K_{a} p^{*}$. The resulting solution is non-iterative. The solution can be expressed in terms of a matrix inverse and is given by $B_{\mathrm{PLS}}=K_{a}\left(K_{a}^{T} X^{T} X K_{a}\right)^{-1} K_{a}^{T} X^{T} Y$ where $K_{a} \in \mathbb{R}^{r \times a}$ is the controllability (Krylov) matrix for the pair $\left(X^{T} X, X^{T} Y\right)$. Relationship to the score and loading vectors are also given in the paper. It is furthermore pointed out that the PLS1 algorithm is equivalent to a truncated Conjugate Gradient (CG) method, Hestenes and Stiefel (1952), for iteratively computing the ordinary least squares solution. Interestingly the PLS1 algorithm is equivalent to a truncated version of Iteration 10.2.13 in Golub and Van Loan (1986), p. 370. Note also the similarity with PLS1 and truncated Lanczos iterations in Algorithm 9.3.1 in Golub and Van Loan (1986), p. 345. This shows that the PLS1 algorithm has strong similarities with iterative methods for solving the normal equation, $X^{T} Y=X^{T} X B$ for the vector $B$ of regression coefficients, in which $X^{T} X$ in this problem is a symmetric matrix. Bi- and tri-diagonalization of symmetric matrices are involved in the iterative LS algorithms. Both the univariate and the multivariate cases are considered in Di Ruscio (2000). The usual PLS2 algorithm for multivariate data presented in the literature is not optimal. A new optimal PLS2 algorithm was also developed along the lines in which the non-iterative PLS1 solution was developed.

\subsubsection{On Subspace System Identification}

A landmark for the development of so called subspace system identification algorithms is the algorithm for obtaining a minimal state space model realization from Hankel matrices constructed from a series of known Markov parameters (or impulse responses), i.e., as presented by Ho and Kalman (1966). This method was completely new to the control community at that time. A numerically efficient implementation of the Ho algorithm through Singular value Decomposition (SVD) was presented in Zeiger and McEwen (1974) and furthermore used to estimate stochastic models directly from observed data in Aoki (1987), and the interest of the topic increased. The DSR algorithm for identifying the entire Kalman filter model matrices directly from observed input and output data was developed in the early 90's and onwards. The method is presented in Di Ruscio $(1996,1994,2003)$ among other papers. The particularly interesting feature of the DSR algorithm is that the Kalman filter gain matrix, $K$, and the square root of the innovation process covariance matrix, $F$, are estimated directly from known input and output data, also documented in Di Ruscio (1996). The innovation process in this algorithm is consistently identified also for closed loop data. A modified implementation of the DSR algorithm which is consistent both for open as well as for closed loop data was developed in the early 2000's and implemented in the dsr_e.m function in the D-SR Toolbox for MATLAB. In this method a series of "future" outputs, $y_{J \mid 1}$, are decomposed into a "signal" part, $y_{J \mid 1}^{d}=D X_{J \mid 1}$, and an innovations ("noise") part, $\varepsilon_{J \mid 1}=F e_{J \mid 1}$ where $e_{k}$ has unit variance, i.e., as

$$
y_{J \mid 1}=y_{J \mid 1}^{d}+\varepsilon_{J \mid 1} .
$$

The decomposition, Equation (1), is consistently computed by projecting the "future" outputs onto the row space of the "past" inputs and outputs, i.e., as

$$
y_{J \mid 1}^{d}=D X_{J \mid 1}=Y_{J \mid 1} /\left[\begin{array}{c}
U_{0 \mid J} \\
Y_{0 \mid J}
\end{array}\right],
$$

where $U_{0 \mid J}$ and $Y_{0 \mid J}$ are defined from "past" inputs and outputs.

Hence, at this stage we have a deterministic identification problem for the entire Kalman filter model matrices, i.e., using that the inputs and output to the Kalman filter are known, i.e., using known "future" inputs $u_{J \mid 1}$ and the known "future" innovations $\varepsilon_{J \mid 1}=y_{J \mid 1}-y_{J \mid 1}^{d}$ as inputs, and using the signal part $y_{J \mid 1}^{d}=D X_{J \mid 1}$ as known outputs. This may efficiently be solved as a deterministic subspace system identification problem in order to estimate the Kalman filter including the system order. Details of this algorithm are presented in Di Ruscio (2008) and used in the PhD thesis work Nilsen (2005). Recently this method, dsr_e.m, is analyzed and compared with the PARSIM-E method by Qin and Ljung (2003); Qin et al. (2005) and it is shown that in general the PARSIM-E method gives larger variance on the parameter estimates compared to dsr_e, which is close to as optimal as the prediction error method. In the PARSIM-E method iterations, 
$i=0,1, \ldots, L$, are used to iteratively compute the future innovations, $\varepsilon_{J \mid 1}, \varepsilon_{J+1 \mid 1}, \cdots, \varepsilon_{J+L \mid 1}$, and at the same time computes Markov parameters as well as a matrix with the same column space as the extended observability matrix. These iterations are believed to give rise to the "high" parameter variance. Notice however that the first step in the PARSIM-E and dsr_e methods are similar. Interestingly Sotomayor et al. (2003) have found the dsr.m algorithm to produce the best model on validation data in comparison with four other subspace methods, CCA, MOESP, N4SID and Robust N4SID. The dsr_e.m algorithm is a variant of the dsr.m algorithm superior for closed loop identification; both dsr.m and dsr_e.m are available in the D-SR Toolbox for MATLAB.

\subsection{Mechanistic Models and Model Based Control}

Modeling and Simulation of Dynamic Systems has been a key course in the education at Telemark University College since 1991; all master students follow this course, and hence it forms a common ground. This course on mechanistic models gives a perfect background for applications of model based control: knowledge of modeling gives a good background for understanding the system under study, and the developed model can be used in a model based controller - or a model that can be used for further simplification. One such control strategy, Model Predictive Control (MPC), has been taught at HiT since 1990, see Lie et al. (2005); Lie and Heath (2008).

Control of polymerization processes was a focus in the 1980-1990s. In Lie (1990), polymerization of polypropylene in a continuous reactor was studied, and the work included a population balance model in the form of moments of the chain length distribution. Part of the work dealt with limitations in attainable bandwidth in optimal control, Lie (1995). Damslora et al. (1998); Damslora (1998) looked into the modeling of a PVC batch reactor, and an optimal control strategy was developed with active use of initiators and inhibitors which indicated the possibility of a significant reduction in the batch time.

Modeling and control of paper production was studied ca. 1999-2009. A simplified model was used to develop an Extended Kalman Filter and the linearized model was used in an MPC algorithm, see Hauge et al. (2005) and Hauge (2003). The solution was implemented at Norske Skog's PM6 in Halden, Norway together with Prediktor in late April 2002. Roger Slora from Norske Skog was instrumental in this project, and also worked with enthusiasm to tailor-make the user interface to something that the operators would accept.
The new control solution was used with success from the beginning of May 2002. A couple of years later, some new measurement equipment was acquired for the paper machine, and this new equipment didn't fit right into the MPC solution. Instead of redesigning the state estimator in the MPC solution, the choice was made to turn off the MPC. This is an interesting observation, and indicates a need to work on the problem of advanced control solutions and how these can be maintained through changes in process, control equipment, and personnel. Later, through the COST E-36 action, Dahlquist (2008), some work was done on model uncertainties and control consequences, Lie (2009).

The production of silicon from ferrosilicon was studied in the period 2000-2004. An advanced population balance model was developed of Elkem's leaching reactor in Bremanger, Norway, and the model was fit to measurements both from laboratory experiments and from operational data, Dueñas Díez et al. (2006). A passivity based nonlinear controller involving reaction networks was developed and tested through simulations. See Dueñas Díez et al. (2008); Dueñas Díez (2004) for details.

In cooperation with the bio engineering group of Professor Rune Bakke at HiT, a Modelica model was developed for the activated sludge purification of water, by fitting a Modelica library for the intended use. A central problem with bio processes is the lack of available measurements, and a study of parameter identifiability was carried out, Sarmiento Ferrero et al. (2006). The possibility to control the system using on-off MPC was studied, with a comparison with simpler control structures, Chai and Lie (2008); Chai (2008). The cooperation with Bakke's group continues through work on biogas production.

Norway has a strong industry in the area of photovoltaic wafers and producing the raw material for these wafers. In cooperation with Elkem, work has been carried out on the solidification of silicon. The process is complicated with two phases and distributed properties within each phase. Two possible modeling strategies are a two domain and a one domain strategy, in both cases leading to relatively nonlinear models, Furenes (2009). In this work, the main idea is to control the solidification rate, as this determines the purity of the final product. The solidification rate is equal to the velocity of the solidification front, which must be inferred from temperature estimates involving nonlinear state estimators. The task is further complicated by few available measurements.

Energy is important for modern society, both the efficient transformation and the efficient use. In a study involving SINTEF Byggforsk and Action 42 in Inter- 
national Energy Agency, a Modelica library is being developed for the climate of buildings, Videla and Lie (2006). The work also involves cogeneration and the use of biofuel in a spark ignition engine, Videla and Lie (2008). Future work will involve activities related to district heating in cooperation with Østfold University College.

As already mentioned elsewhere in this edition of MIC, some past work with Xstrata on $\mathrm{Cu}$ leaching, Lie and Hauge (2008); Alic et al. (2009.), will continue and will be extended. Also, some activity in the production of silicon for the PV industry is ongoing, Komperød et al. (2009).

\subsection{Nonlinear Model Predictive Control of Polyolefin Plants}

Linear Model Predictive Control (MPC) such as the DMC algorithm has become popular in plants such as oil refineries and crackers during the last few decades. In polyolefin production, linear MPC has been tested with only limited success and is not widely used. The perhaps most important reason for this limited success is that a typical polyolefin plant operates over a wide operation window to produce products of different qualities. Thus, the inherent non-linearities of such processes become evident, and good control with a linear control scheme will be difficult.

During the last decade, nonlinear MPC has been successfully implemented in many polyolefin plants. The first known implementation of nonlinear MPC in an industrial plant was done by Borealis on a polypropylene plant in Norway. This controller was put into closedloop in 1993 and (upgraded versions) has been used continuously until the plant was shut down a couple of years ago. Borealis' technology for nonlinear MPC has proven to be successful through the implementation in practically all Borealis polyolefin plants and is also an integral part of Borealis' Borstar technology, Glemmestad and Hillestad (2001), Glemmestad et al. (2004), for polyolefin production built in Europe, the Middle East and Asia.

During the last years, commercial technology for nonlinear MPC has also become available and implemented in many polyolefin plants. While Borealis uses mathematical models based on first principles, some commercial vendors of nonlinear MPC use models based on artificial neural networks or nonlinear statespace models based on plant responses etc.

The process model used by the nonlinear MPC technology in Borealis is a nonlinear state space model that roughly can be divided into the following parts:

- Dynamic mass balance equations $(\mathrm{dm} / \mathrm{dt}=$ inflow - outflow - reacted).
- Reaction kinetics (also aggregated to calculate production rate and split factors).

- Polymer property calculations (e.g., Melt Flow Rate).

- Some thermodynamic calculations.

- Calculation of various plant measurements (for online model updating).

One advantage of first principles modeling is that the models can be reused. That is, the modeling in a new project does not start from scratch but instead one can start with the best knowledge from previous projects. Each mass balance equation is usually quite simple to create, however, knowledge and experience is needed in order to select states to model and what can be omitted in the model. Reaction kinetics modeling is usually done based on lab experiments but will normally also be tuned against plant data. The control problem is solved using an SQP algorithm and the parametrization of the control signal is flexible.

Figure 5 shows results from a critical transition in a real plant before and after the nonlinear MPC (called OnSpot) is installed, Glemmestad et al. (2002). Thick lines are with OnSpot and thin lines are without OnSpot. Hydrogen concentration is shown in the upper plot, production rate in the middle and solid concentration is in the lower plot. The results demonstrate that nonlinear MPC yields a faster transition, but first of all it yields higher production rate (plot in middle) and a safer operation due to less variation in the solids concentration in the reactor (lower plot).

The success of nonlinear MPC in the polyolefin area, Haugwitz et al. (2008), shows that linear MPC is not always sufficient for satisfactory control and that nonlinear MPC now is becoming a mature technology within some industry segments.

\section{PhD Studies}

An important part of research is work with $\mathrm{PhD}$ students. Through the years, 17 candidates have defended their thesis through the cooperation with NTNU.

1. Olav Aaker, Aaker (1996). Disputation at Telemark University College, October 30 1996. Main supervisor: Jens I. Ytreeide.

2. Bjørn Glemmestad, Glemmestad (1997). Disputation at Telemark University College, December 12 1997. Main supervisor: Truls Gundersen.

3. André Johan Damslora, Damslora (1998). Disputation at Telemark University College, May 11 1998. Main supervisor: Bernt Lie. 

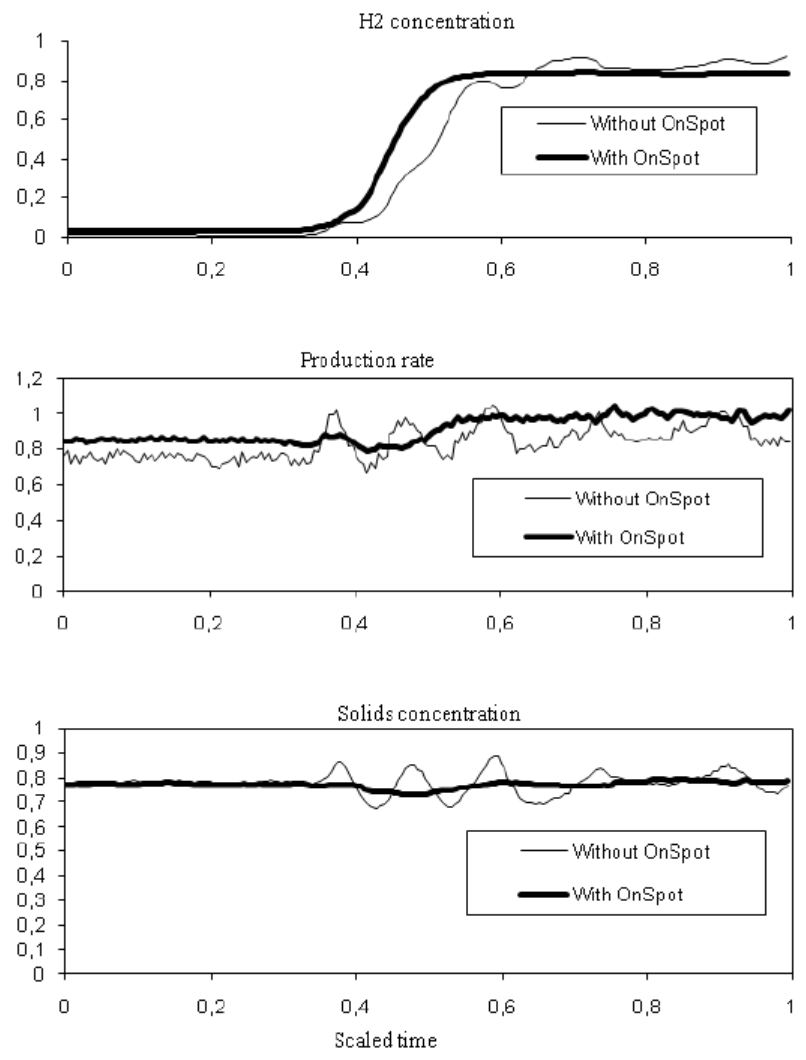

Figure 5: Results from a critical transition in a real plant before (thin lines) and after (thick lines) installation of OnSpot. Scaled variables are shown, with hydrogen concentration (top), production rate (middle), and solids concentration (bottom).

4. Rolf Ergon, Ergon (1999a). Disputation at Telemark University College, October 1 1999. Main supervisor: Jens I. Ytreeide.

5. Thorbjørn Tønnesen Lied, Lied (2000). Disputation at Telemark University College, February 9 2001. Main supervisor: Kim Esbensen.

6. Juan Huang, Huang (2001). Disputation at Telemark University College, May 22 2001. Main supervisor: Kim Esbensen.

7. Maths Halstensen, Halstensen (2001). Disputation at Telemark University College, January 14 2002. Main supervisor: Kim Esbensen.

8. Maria Lundhaug, Lundhaug (2002). Disputation at Telemark University College, April 292002. Main supervisor: Kim Esbensen.

9. Tor Anders Hauge, Hauge (2003). Disputation at Telemark University College, May 8 2003. Main supervisor: Bernt Lie.

10. Marta Dueñas Díez, Dueñas Díez (2004). Disputation at Telemark University College, May 282004. Main supervisor: Bernt Lie. Dueñas Díez was the recipient of the first European EFCE Excellence Award in Recognition of Outstanding PhD Thesis in the area of CAPE, awarded in 2006.

11. Geir Werner Nilsen, Nilsen (2005). Disputation at Telemark University College, June 27 2006. Main supervisor: David Di Ruscio.

12. Olav Gerhard Haukenes Nygaard, Nygaard (2006). Disputation at Telemark University College, October 5 2006. Main supervisor: Saba Mylvaganam.

13. Kjell Joar Alme, Alme (2007). Disputation at Telemark University College, December 122007. Main supervisor: Saba Mylvaganam.

14. Urmila Datta, Datta (2007). Disputation at Telemark University College, January 17 2008. Main supervisor: Saba Mylvaganam.

15. Qian Chai, Chai (2008). Disputation at Telemark University College, May 20 2008. Main supervisor: Bernt Lie.

16. Nils-Olav Skeie, Skeie (2008). Disputation at Telemark University College, October 16 2008. Main supervisor: Bernt Lie.

17. Beathe Furenes, Furenes (2009). Disputation at Telemark University College, December 92009. Main supervisor: Bernt Lie.

More candidates are in the pipeline, and while the contact with NTNU will remain important, future candidates will mainly be associated with HiT's own program in Process, Energy, and Automation Engineering. Multidisciplinary problems with industrial relevance will be studied in cooperation with the Department of Process, Energy, and Environment Technology at HiT, and with the industry.

\section{Activities in Societies}

NFA - the Norwegian Federation of Automatic Control: Finn Haugen has been a frequent contributor with industrially oriented courses. The Norwegian chapter of SIMS - the Scandinavian Simulation Society - is organized in NFA. Bernt Lie has served in the board of SIMS for a decade, and is an active participant in SIMS conferences. 
Modelica ${ }^{\circledR}$ _ The free modeling language Modelica ${ }^{\circledR}$ is developed by the Modelica Association, a non-profit organisation with members from industry and academia. Dietmar Winkler has been an active member of the Modelica Association for several years now. To participate actively in the development of open source Modelica ${ }^{\circledR}$ tools the group has become an organizational member of The Open Source Modelica Consortium (OSMC) in October 2009.

IET - the Institution of Engineering and Technology: Saba Mylvaganam has close collaboration with IET, and has jointly organized many seminars and workshops during the last decade. Through the IET, lecture tours have been organized in Norway with prominent industrialists and academics from the UK. tel-tek — Telemark Teknisk Industrielle Utviklingssenter: Saba Mylvaganam and Maths Halstensen have close collaboration with the R\&D organisation tel-tek in Porsgrunn. There have been and are many activities running in collaboration with tel-tek, funded by the Research Council of Norway and the industry.

$\mathrm{EU}$ - the group has been involved in several EU-thematic projects such as THEIERE, EIESurveyor,and is currently involved in ELLEIEC, and have developed different modules for teaching purposes. Under the period of the THEIERE and EIESurveyor, the group was involved as coordinator for the Measurement and Control module, particularly the block on "Sensor to Web", where our flowrings were used for demonstrations.

Nordic Process Control Group — Bjørn Glemmestad and Bernt Lie have served in the board. Lie has organized their workshop in 2009.

\section{References}

Aaker, O. Operator Support and Diagnostic Reasoning in an Industrial Process. Ph.D. thesis, NTNU, Porsgrunn, 1996. NTNU-HiT, 82-7119-976-5, 0802-3271.

Alic, M., Hauge, T. A., and Lie, B. Developing A Simple Modelica Library For Simulation of The Xstrata Nikkelverk Copper Production Plant. In Dong Energy, editor, Proc. 50th Scand. Conf. on Simulation and Modeling (SIMS). Fredericia, Denmark, 2009.

Alme, K. J. Artificial Neural Network for Direct Parameter Estimation in Electrical Capacitance Tomography. In Protocom 2006. Warsawa, 2006.

Alme, K. J. Material Distribution and Interface Detection Using EIT. Ph.D. thesis, NTNU-HiT, Porsgrunn, 2007. 2007:223, ISBN 978-82-471-4929-3 (printed), 978-82-471-4932-0 (elec.), ISSN 1503-8181.
Alme, K. J. and Mylvaganam, S. Conductivity Effects in Electrical Tomography Systems with focus on 3D Effects Using COMSOL Multiphysics Modules. In Comsol Multiphysics Conf. Copenhagen, 2006a.

Alme, K. J. and Mylvaganam, S. Electrical Capacitance Tomography - Sensor Models, Design, Simulations and Experimental Verification. IEEE Sensors Journal, 2006b. 6(5):1256-1266. doi:10.1109/JSEN.2006.881409.

Alme, K. J. and Mylvaganam, S. Comparison of Different Measurement Protocols in Electrical Capacitance Tomography Using Simulations. IEEE Trans. on Instr. \& Meas., 2007. 56(6):2119-2130.

Aoki, M. State Space Modeling of Time Series. Springer-Verlag Berlin Heidelberg, 1987.

Chai, Q. Modeling, Estimation, and Control of Biological Wastewater Treatment Plants. Ph.D. thesis, NTNU-HiT, Porsgrunn, 2008. 2008:108, ISBN 978-82471-8155-3, 978-82-471-8169-0, ISSN 1503-8181.

Chai, Q. and Lie, B. Predictive Control of an Intermittently Aerated Activated Sludge Process. American Control Conference, 2008. June:11-13. Seattle, USA.

Dahlquist, E. Use of Modeling and Simulation in Pulp and Paper Industry. 2008. URL http://works. bepress.com/dr_erik_dahlquist. ISBN-977493-05 .

Damslora, A. J. Optimisation Based Control of Batch Emulsion Polymerisation of Vinyl Chloride. Ph.D. thesis, NTNU-HiT, Porsgrunn, 1998. Thesis 1998:29, ISBN 82-471-0224-2.

Damslora, A. J., Lie, B., and Sælid, S. Reduction of PVC Batch Time by Optimal Control of Free Radical Concentration. Nonlinear Model Based Control, 1998. 353:781-803. Kluwer, The Netherlands.

Datta, U. Multimodal Measurements in Dilute Phase Pneumatic Conveying Systems. Ph.D. thesis, NTNU-HiT, 2007. 2007:240, ISBN 978-82-471-5322-2 (printed), 978-82-471-5336-9 (elec.), ISSN 1503-8181.

Datta, U., Dyakowski, T., and Mylvaganam, S. Average Particle Size and Instantaneous Mass Flow Rate Estimation in Dilute Phase Pneumatic Conveying Systems using ECT. In 5th World Congress on Industrial Process Tomography. Bergen, 2007a.

Datta, U., Dyakowski, T., and Mylvaganam, S. Estimation of Particulate Velocity Components in Pneumatic Transport Using Pixel Based Correlation with Dual Plane ECT. Chemical Engineering Journal, 2007b. 130(2/3):87-99. doi:10.1016/j.cej.2006.08.034. 
Datta, U., Mathiesen, V., and Mylvaganam, S. Tomometric Approach using Multi Sensor Data Fusion in Particle Segregation Studies. In 3rd World Cong. on Industrial Process Tomography. Banff, Canada, 2003.

Di Ruscio, D. Methods for the Identification of State Space Models from Input and Output Measurements. In SYSID 94, The 10th IFAC Symposium on System Identification. Copenhagen, 1994.

Di Ruscio, D. Combined Deterministic and Stochastic System Identification and Realization: DSR - a Subspace Approach Based on Observations. Modeling, Identification and Control, 1996. 17(3):193-230. doi:10.4173/mic.1996.3.3.

Di Ruscio, D. A Weighted View of the Partial Least Squares Algorithm. Automatica, 2000. 36:831-850. doi:10.1016/S0005-1098(99)00210-1.

Di Ruscio, D. Subspace System Identification of the Kalman Filter. Modeling, Identification and Control, 2003. 24(3):125-157. doi:10.4173/mic.2003.3.1.

Di Ruscio, D. Subspace System Identification of the Kalman Filter: Open and Closed Loop Systems. In Proc. of the Intl. Multi-Conference on Engineering and Technological Innovation. Orlando, USA, 2008.

Dueñas Díez, M. Population Balance Modeling and Passivity-based Control of Particulate Processes, Applied to the Silgrain ${ }^{\circledR}$. Ph.D. thesis, NTNUHiT, Porsgrunn, 2004. 2004:35, ISBN 82-471-6276-8 (printed), 82-471-6274-1 (elec.), ISSN 1503-8181.

Dueñas Díez, M., Fjeld, M., Andersen, E., and Lie, B. Validation of a Compartmental Population Balance Model of an Industrial Leaching Process: The Silgrain ${ }^{\circledR}$ Process. Chemical Engineering Science, 2006. 61(1):229-245. doi:10.1016/j.ces.2005.01.047.

Dueñas Díez, M., Ydstie, B. E., Fjeld, M., and Lie, B. Inventory Control of Particulate Processes. Computers and Chemical Engineering, 2008. 32:46-67. doi:10.1016/j.compchemeng.2007.01.007.

Ergon, R. Dynamic System Multivariate Calibration for Optimal Primary Output Estimation. Ph.D. thesis, NTNU, Trondheim, 1999a. 1999:72, NTNU-HiT, ISBN 82-471-0442-3, ISSN 0802-3271.

Ergon, R. On primary output estimation by use of secondary measurements as input signals in system identification. IEEE Trans. Autom. Control, 1999b. 44(4):821-825. doi:10.1109/9.754826.
Ergon, R. Noise handling capabilities of multivariate calibration models. Modeling, Identification and Control, 2002a. 23:259-273. doi:10.4173/mic.2002.4.2.

Ergon, R. PLS score-loading correspondence and a biorthogonal factorization. J. Chemometrics, 2002b. 16(7):368-373. doi:10.1002/cem.736.

Ergon, R. Compression into two-component PLS factorization. J. Chemometrics, 2003. 17(6):303-312. doi:10.1002/cem.803.

Ergon, R. Informative PLS score-loading plots for process understanding and monitoring. Process Control, 2004. 14(8):889-897. doi:10.1016/j.jprocont.2004.02.004.

Ergon, R. PLS post-processing by similarity transformation (PLS+ST): A simple alternative to 0PLS. Journal of Chemometrics, 2005. 19(1):1-4. doi:10.1002/cem.899.

Ergon, R. Reduced PCR/PLSR models by subspace projection. Chemom. Intell. Lab. Syst., 2006. 81(1):68-73. doi:10.1016/j.chemolab.2005.09.008.

Ergon, R. Finding Y-relevant part of X by use of PCR and PLSR model reduction methods. Journal of Chemometrics, 2007. 21:537-546. doi:10.1002/cem.1062.

Ergon, R. Informative score-loading-contribution plots for multi-response process monitoring. Chemom. Intell. Lab. Syst., 2009a. 95(1):31-34. doi:10.1016/j.chemolab.2008.08.001.

Ergon, R. Re-interpretation of NIPALS results solves PLSR inconsistency problem. Journal of Chemometrics, 2009b. 23:72-75. doi:10.1002/cem.803.

Ergon, R. and Di Ruscio, D. Dynamic system calibration by system identification methods. In Fourth European Control Conf. (ECC 97). Brussels, 1997.

Ergon, R. and Halstensen, M. Dynamic system multivariate calibration based on multirate sampling data. Modeling, Identification and Control, 2001. 22(2):73-88. doi:10.4173/mic.2001.2.2.

Esbensen, K. Multivariate Analysis - in practice. Camo AS, Oslo, 5th edition, 2001.

Esbensen, K. H., Halstensen, M., Lied, T. T., Saudland, A., Svalestuen, J., de Silva, S., and Hope, B. Acoustic chemometrics - from noise to information. Chemom. Intell. Lab. Sys., 1998. 44:61-76. doi:10.1016/S0169-7439(98)00114-2. 
Esbensen, K. H., Hope, B., Lied, T. T., Halstensen, M., Sundberg, K., and Gravermoen, T. Acoustic chemometrics for fluid flow quantifications -II: A small constriction will go a long way. Journal of Chemometrics, 1999. 13:209-236.

Furenes, B. Model Based Control of Solidification. Ph.D. thesis, NTNU-HiT, 2009. 2009:228, ISBN-4711860-3, 978-82-471-1861-0, ISSN 1503-8181.

Glemmestad, B. Optimal Operation of Integrated Processes, Studies on heat recovery systems. Ph.D. thesis, NTNU-HiT, 1997. 1997:118, ISBN 82-471-0156-4, ISSN 0802-3271.

Glemmestad, B., Ertler, G., and Hillestad, M. Advanced Process Control in a Borstar PP Plant. In Proc. from ECOREP II. Lyon, pages 46-49, 2002.

Glemmestad, B. and Hillestad, B. Experience from an Industrial MPC Implementation. In NPC10. NPC, Åbo, Finland, pages 77-78, 2001.

Glemmestad, B., Hofsten, K., Wilsher, E., and Andersen, K. S. Non-Linear Model Predictive Control of Polyolefin Plants. In NPC12. n/a, Gothenburg, Sweden, pages 66-67, 2004.

Golub, G. and Kahan, W. Calculating the singular values and pseudo-inverse of a matrix. SIAM J. Numer. Anal., 1965. 2:205-224.

Golub, G. H. and Van Loan, C. F. Matrix Computations. North Oxford Academic, London, 1986.

Halstensen, M. Acoustic Chemometrics. Experimental multivariate sensor technology and development of system prototypes for industrial multi-phase characterisation: selected forays. Ph.D. thesis, NTNU-HiT, 2001. 2001:111, ISBN 82-471-5381-5, ISSN 0809-103X.

Halstensen, M., de Bakker, P., and Esbensen, K. H. Acoustic chemometric monitoring of an industrial granulation production process - a PAT feasibility study. Chemom. Intell. Lab. Syst., 2006. 84:88-97. doi:10.1016/j.chemolab.2006.05.012.

Halstensen, M. and Esbensen, K. H. New developments in acoustic chemometric prediction of particle size distribution - the problem is the solution. Journal of Chemometrics, 2000. 14:463-481.

Halstensen, M., de Silva, S., and Esbensen, K. H. Acoustic monitoring of pneumatic transport lines from noise to information. KONA Powder and Particle, 1998. 16:170-178.

Hauge, T. A. Roll-out of Model Based Control with Application to Paper Machines. Ph.D. thesis, NTNU, 2003. 2003:31, ISBN 82-471-5581-8, ISSN 0809-103X.
Hauge, T. A., Slora, R., and Lie, B. Application and roll-out of infinite horizon MPC employing a nonlinear mechanistic model to paper machines. Journal of Process Control, 2005. 15(2):201-213. doi:10.1016/j.jprocont.2004.05.003.

Haugen, F. Introduction to LabVIEW Control Design, System Identification and Simulation Tools. In $\mathrm{Na}$ tional Instruments Days. Drammen, Norway, 2005.

Haugen, F. Examples of Student Assignments on Modeling, Simulation, and Control. In National Instruments Days. Drammen, Norway, 2008.

Haugen, F. Air Heater. Technical report, Telemark University College, 2009a. URL http://home.hit. no/ finnh/air_heater.

Haugen, F. Basic Dynamics and Control. TechTeach, 2009b. ISBN 978-82-91748-13-9.

Haugen, F. Lab assignment: Hardware-in-the-loop (HIL) simulation. 2009c. URL http://www2.hit. no/tf/fag/sce2006/2009/hil_sim.

Haugen, F. Lab assignment: Implementation of a control system. 2009d. URL http://www2.hit.no/tf/ fag/sce1106/2009/project/project.

Haugen, F. Lab assignment: Model-based predictive control (MPC). 2009e. URL http://www2.hit.no/ $\mathrm{tf} / \mathrm{fag} / \mathrm{scev3106/2009/projects/mpc}$.

Haugen, F. Lab assignment: Soft-sensor (state estimator). 2009f. URL http://www2.hit.no/tf/fag/ sce4206/2009/softsensor.

Haugen, F. Lab assignment: System identification. 2009g. URL http://www2.hit.no/tf/fag/ sce4206/2009/system_ident.

Haugen, F. Lecture Notes in Models, Estimation and Control. TechTeach, 2009h. ISBN 978-82-91748-146.

Haugen, F. Water Tank. Technical report, Telemark University College, 2009i. URL http://home.hit. no/ finnh/dok_tankmodell.

Haugen, F., Fjelddalen, E., Edgar, T., and Dunia, R. Examples of Student Assignments on Modeling, Simulation, and Control. CACHE News (Computer Aids for Chemical Engineering), 2007. Winter.

Haugen, F., Fjelddalen, E., Edgar, T., and R.Dunia. A Complete Programming Framework for Process Control Education. In 2nd IEEE Multi-conference on Systems and Control. IEEE, San Antonio, 2008. 
Haugwitz, S., Wilsher, E., Hofsten, K., Andersen, K. S., and Glemmestad, B. Commissioning of Nonlinear Model Predictive Controllers to a NewPolypropylene Plant. In Reglermötet. Sweden, 2008.

Hestenes, M. R. and Stiefel, E. Methods for Conjugate Gradients for Solving Linear Systems. J. of Res. of the national Bureau of Stand., 1952. 49(6):409-436.

Ho, B. L. and Kalman, R. E. Effective construction of linear state-variable models from input/output functions. Regelungstechnik, 1966. 14(12):545-592.

Huang, J. Developments in Applied Chemometrics $A M T$, acoustic chemometrics and $N$-way image analysis. Ph.D. thesis, NTNU-HiT, Porsgrunn, 2001. 2001:25, ISBN 82-7984-187-3, ISSN 0809-103X.

Komperød, M., Bones, J. A., and Lie, B. Solution to an Implementation Issue for a Two-Step ARX Algorithm, with Application to the Czochralski Crystallization Process. In Proc. 50th Scand. Conf. on Simulation and Modeling. Fredericia, 2009.

Lie, B. Control Structures for Polymerization Processes Applied to Polypropene Manufacturing. Ph.D. thesis, NTNU, Trondheim, 1990. Report 82-W.

Lie, B. Attainable Performance in LQG Control. Methods of Model Based Process Control, 1995. 293:263295. Kluwer Academic Publishers.

Lie, B. Model Uncertainty and Control Consequences: a Paper Machine Study. Mathematical and Computer Modelling of Dynamical Systems, 2009. 15(5):463-477. doi:10.1080/13873950903375452.

Lie, B., Dueñas Díez, M., and Hauge, T. A. A Comparison of Implementation Strategies for MPC. Modeling, Identification and Control, 2005. 26(1):39-50. doi:10.4173/mic.2005.1.3.

Lie, B. and Hauge, T. A. Modeling of an Industrial Copper Leaching and Electrowinning Process, with Validation Against Experimental Data. In Proc. 49th Scand. Conf. on Simulation \& Modeling. Oslo, 2008.

Lie, B. and Heath, W. Model Based Control. In E. Dahlquist, editor, Use of Modeling and Simulation in Pulp and Paper Industry. pages 978-91, 2008. URL http://works.bepress.com/dr_erik_ dahlquist. (C) COST Office, ISBN-977493-0-5.

Lied, T. T. Multivariate Image Regression (MIR) for Quantitative Predictions. Ph.D. thesis, NTNU-HiT, 2000. 2000:99, ISBN 82-7984-126-1, ISSN 0809-103X.

Lorentzen, H., Timmerberg, J., and Mylvaganam, S. Calculation of Cable Parameters for Different Cable Shapes. In European COMSOL Conf. 2008.
Lundhaug, M. Sea Ice Studies in the Northern Sea Route by Use of Synthetic Aperture Radar. Ph.D. thesis, NTNU-HiT, Porsgrunn, 2002. 2002:22, ISBN 82-471-5414-5, ISSN 0809-103X.

Martens, H. and Næs, T. Multivariate Calibration. John Wiley and Sons Ltd, 1989.

Modelica-Association. Modelica ${ }^{\circledR}$ - A Unified ObjectOriented Language for Physical Systems Modeling Language Specification. Modelica Association, version 3.1 edition, 2009. URL http://www . modelica. org.

Modelica.org. Tools page on Modelica.org. 2009. URL http://www.modelica.org/tools.

Mylvaganam, S. Some Applications of Acoustic Emission in Particle Science and Technology. Particulate Science and Technology, 2003. 21(3):293-301. doi:10.1080/02726350307485.

Mylvaganam, S., Datta, U., Halstensen, M., and Mathiesen, V. Multi Sensor Suite Comprising Active and Passive Transducers for Monitoring Solids Flow and Silo Integrity. In IEEE UFFC Symp. Honolulu, 2003.

Mylvaganam, S. and Dyakowski, T. Estimating transverse velocity components of slug flow using pixel by pixel correlation method using ECT. In 4th World Cong. Ind. Proc. Tomography. Aizu, pages 5-8, 2005.

Nilsen, G. W. Topics in Open and Closed Loop Subspace System Identification: Finite Databased Methods. Ph.D. thesis, NTNU-HiT, 2005. 2005:228, ISBN7357-3, 82-471-7356-5, ISSN 1503-8181.

Nygaard, G., Nævdal, G., and Mylvaganam, S. Evaluating Nonlinear Kalman Filters for Parameter Estimation in Reservoirs During Petroleum Well Drilling. In IEEE Conference on Control Applications. München, Germany, pages 4-6, 2006.

Nygaard, G. H. and Nævdal, G. Non-linear model predictive control scheme for stabilizing annulus pressure during oil well drilling. Journal of Process Control, 2006. 16(7):719-732. doi:10.1016/j.jprocont.2006.01.002.

Nygaard, G. H., Vefring, E. H., Fjelde, K.-K., Nævdal, G., Lorentzen, R. J., and Mylvaganam, S. Bottomhole Pressure Control during Drilling Operations in Gas-Dominant Wells. SPE Journal, 2007. 12(1):4961. doi:10.2118/91578-PA.

Nygaard, G. H., Vefring, E. H., Lorentzen, R. J., Nævdal, G., Fjelde, K. K., and Mylvaganam, S. Bottomhole pressure control during pipe connection in 
gas-dominant wells. In $S P E / I A D C$ Underbalanced Tech. Conf. and Exhibition. Houston, 2004a.

Nygaard, G. H., Vefring, E. H., Mylvaganam, S., Lorentzen, R. J., Nævdal, G., and Fjelde, K. K. Underbalanced drilling: improving pipe connection procedures using automatic control. In SPE Ann. Tech. Conf. and Exh. Conv. Center, Houston, 2004b.

Nygaard, O. G. H. Multivariable Process Control in High Temperature and High Pressure Environment Using Non-intrusive Multi Sensor Data Fusion. Ph.D. thesis, NTNU-HiT, 2006. 2006:38, ISBN 82-471-7820-6, 82-471-7819-2, ISSN 1503-8181.

Pell, R., Ramos, L., and Manne, R. The model space in partial least squares regression. Journal of Chemometrics, 2007. 21:165-172. doi:10.1002/cem.1067.

Qin, S. J. and Ljung, L. Closed-loop Subspace Identification with Innovation Estimation. In Proc. of the 13th IFAC SYSID Symposium. pages 887-892, 2003.

Qin, S. J., Weilu, L., and Ljung, L. A Novel Subspace Approach with Enforced Causal Models. Automatica, 2005. 41:2043-2053. doi:10.1016/j.automatica.2005.06.010.

Sarmiento Ferrero, C., Chai, Q., Dueñas Díez, M., Amrani, S. H., and Lie, B. Systematic Analysis of Parameter Identifiability for Improved Fitting of a Biological Wastewater Model to Experimental Data. Modeling, Identification and Control, 2006. 27(4):219-238. doi:10.4173/mic.2006.4.2.

Skeie, N.-O. Soft Sensors for Level Estimation. Ph.D. thesis, NTNU-HiT, Porsgrunn, 2008. 2008:102, ISBN 978-82-471-4462-6, 978-82-471-4476-3, ISSN 1503-8181.

Skeie, N.-O. and Lie, B. Level and Interface Estimation in an Oil / Water Separator Using Multi Sensor Data Fusion, in E. In E. Juuso, editor, 47th Conf. on Simulation and Modelling. pages 210-215, 2006.

Skeie, N.-O., Mylvaganam, S., and Lie, B. Using Multi Sensor Data Fusion for Level Estimation in a Separator, in W. In 16th European Symp. on Comp. Aided Proc. Engn. and 9th Intl. Symp. on Proc. Sys. Engn., volume 21. Elsevier, pages 1383-1388, 2006.

Sotomayor, O. A. Z., Parka, S. W., and Garcia, C. Multivariable Identification of an Activated Sludge Process with Subspace-based Algorithms. Control Engineering Practice, 2003. 11:961-969. doi:10.1016/S0967-0661(02)00210-1.

Timmerberg, J., Beckmann, P., and Mylvaganam, S. Quasianalytical Estimates of Inductance Using Subconductor Methods. In Intern. Conference: Environment, Technology, Resources. Rezekne, Latvia, 2009.
Trygg, J. and Wold, S. Orthogonal projections to latent structures. O-PLS. J. Chemometrics, 2002. 16:119128. doi:10.1002/cem.695.

Vefring, E. H., Nygaard, G. H., Fjelde, K. K., Rolf, Lorentzen, J., Nævdal, G., and Merl, A. Reservoir Characterization During Underbalanced Drilling: Methodology, Accuracy, and Necessary Data. In Proc. SPE Ann. Tech. Conf. San Antonio, 2002.

Videla, J. I. and Lie, B. A New Energy Building Simulation Library. In C. Kral and A. Haumer, editors, Proc. of the 5th Intl. Modelica Conference. The Modelica Association, Vienna, pages 685-693, 2006.

Videla, J. I. and Lie, B. Model Reduction of a Micro ICE Based CHP Model. In 1st Intl. Conf./Works. in Micro-Cogeneration Techn. and Appl. 2008.

Viumdal, H., Ru, B., Y.and Moxnes, and Liane, S., M. an Mylvaganam. Multi sensor data fusion for aluminium cell health monitoring and control. In TMS annual meeting Proceedings CD. Seattle, 2010.

Wærstad, H., Cortvriend, L., Datta, U., Mathiesen, V., and Mylvaganam, S. Multi Sensor Data Fusion With Flange Mounted Acoustic Emission Sensors In The Monitoring Of Fluidised Beds. In IEEE UFFC Symposium. Munich, Germany, 2002.

Winkler, D. and Gühmann, C. Simulation of Electric Drives using freeFOClib. In Proc. of IEEE Intl. Conf. on Sustainable Energy Tech. IEEE, SMU Conf. Centre, Singapore, 2008. doi:10.1109/ICSET.2008.4747173.

Winkler, D. and Gühmann, C. Simulation of Faults in Electric Drive Systems with Modelica. In Proc. MATHMOD 09 Vienna. 2009.

Yahoui, A., Mouhoumed, B., Ducharne, B., Mylvaganam, S., and Sixdenier, F. Diagnostic of interturn defect in three phase system by studying hysteresis magnetic harmonics signatures. In Proc. ICEM 2004, XVI Intl. Conf. On Electrical Mach. 2004.

Yahoui, H. and Mylvaganam, S. Modelling and characterisation of hysteresis phenomena in 3-phase power systems for diagnostic purposes. In Symp. on Diagfor Electrical Mach., Power Elec. and Drives. 2009.

Zeiger, H. and McEwen, A. Approximate Linear Realizations of Given Dimensions Via Ho's Algorithm. In IEEE Trans. on Automatic Control. 1974. doi:10.1109/TAC.1974.1100525. 